\title{
PF\#11-0408
}

\section{A thin rivulet or ridge subject to a uniform transverse shear stress at its free surface due to an external airflow}

\author{
J. M. Sullivan, C. Paterson, S. K. Wilson*, and B. R. Duffy \\ Department of Mathematics and Statistics, University of Strathclyde, \\ 26 Richmond Street, Glasgow G1 $1 X H$, UK
}

(Dated: 30th March 2009, revised 18th May 2011, 8th June 2012 and 11th July 2012)

\footnotetext{
* Author for correspondence. Presently also a Visiting Fellow in the Oxford Centre for Collaborative Applied Mathematics (OCCAM), University of Oxford, Mathematical Institute, 24-29 St. Giles', Oxford OX1 3LB. Email: s.k.wilson@strath.ac.uk, Telephone: + 44 (0) 141548 3820, Fax: + 44 (0) 141548 3345 .
} 


\begin{abstract}
We use the lubrication approximation to analyse three closely related problems involving a thin rivulet or ridge (i.e. a two-dimensional droplet) of fluid subject to a prescribed uniform transverse shear stress at its free surface due to an external airflow, namely a rivulet draining under gravity down a vertical substrate, a rivulet driven by a longitudinal shear stress at its free surface, and a ridge on a horizontal substrate, and find qualitatively similar behaviour for all three problems. We show that, in agreement with previous numerical studies, the free surface profile of an equilibrium rivulet/ridge with pinned contact lines is skewed as the shear stress is increased from zero, and that there is a maximum value of the shear stress beyond which no solution with prescribed semi-width is possible. In practice, one or both of the contact lines will de-pin before this maximum value of the shear stress is reached, and so we consider situations in which the rivulet/ridge de-pins at one or both contact lines. In the case of de-pinning only at the advancing contact line, the rivulet/ridge is flattened and widened as the shear stress is increased from its critical value, and there is a second maximum value of the shear stress beyond which no solution with a prescribed advancing contact angle is possible. In contrast, in the case of de-pinning only at the receding contact line, the rivulet/ridge is thickened and narrowed as the shear stress is increased from its critical value, and there is a solution with a prescribed receding contact angle for all values of the shear stress. In general, in the case of de-pinning at both contact lines there is a critical "yield" value of the shear stress beyond which no equilibrium solution is possible and the rivulet/ridge will evolve unsteadily. In an Appendix we show that an equilibrium rivulet/ridge with prescribed flux/area is quasi-statically stable to two-dimensional perturbations.
\end{abstract}




\section{INTRODUCTION}

Films, rivulets and droplets of fluid subject to various external airflows occur in many situations ranging from the everyday (such as, for example, rainwater on the windows of a moving vehicle) to engineering applications such as in ice accretion on aircraft wings (see, for example, Myers and Charpin ${ }^{1}$ ), in air-knife and spin-coating processes (see, for example, Chou and $\mathrm{Wu}^{2}$ ), and even in the rain-wind induced vibrations of the cables of cable-stayed bridges (see, for example, Robertson et al. ${ }^{3}$ ). As a result, there has been a significant amount of both theoretical and experimental research into the behaviour of fluid films, rivulets and droplets subject to external pressure and/or surface-shear-stress effects (see, for example, Fan, Wilson and $\operatorname{Kapur}^{4}$ ). In particular, there has been considerable interest in the critical "yield" value of the shear stress beyond which a droplet on a substrate cannot remain at a fixed location but is displaced along it, perhaps ultimately becoming completely detached from it.

In their pioneering work Li and Pozrikidis ${ }^{5}$, Dimitrakopoulos and Higdon ${ }^{6,7}$, Schleizer and Bonnecaze $^{8}$, Yon and Pozrikidis ${ }^{9}$, and Dimitrakopoulos ${ }^{10}$ used boundary-integral methods to study either a two-dimensional or a three-dimensional droplet on a planar substrate in a shear flow. The effects of fluid inertia were investigated for a two-dimensional droplet by Zhang, Miksis and Bankoff ${ }^{11}$ using a front-tracking method and for a three-dimensional droplet by Spelt ${ }^{12}$ using a level-set method and by Ding and Spelt ${ }^{13}$ and Ding, Gilani and Spelt ${ }^{14}$ using a diffuse-interface method. Depending on the details of the specific problem considered, these authors demonstrated and quantified initial deformation possibly followed by subsequent de-pinning, sliding, pinch-off, and even perhaps complete detachment of the droplet from the substrate as the strength of the shear flow is increased. Researchers have also used approximate and/or asymptotic approaches which complement and help to elucidate the results of these numerical investigations. For example, King and Tuck ${ }^{15}$ used thin-aerofoil theory to analyse the possible equilibrium solutions for a thin two-dimensional droplet supported against gravity on an inclined planar substrate by an external air flow. More recently, Sugiyama and Sbragaglia ${ }^{16}$ obtained a series solution for a hemispherical droplet in a shear flow and, in particular, used it to obtain an approximate solution for a weakly deformed droplet. In her pioneering work Dussan V. ${ }^{17}$ used the lubrication ap- 
proximation together with the additional assumption that the contact-angle hysteresis (i.e. the difference between the advancing and the retreating contact angles) is much smaller than the retreating contact angle to obtain an approximate expression for the critical value of the shear stress for a thin three-dimensional droplet, and Dimitrakopoulos and Higdon ${ }^{6}$ (Appendix) obtained the corresponding result for a thin two-dimensional droplet. However, perhaps unsurprisingly in view of the rather restrictive assumption they made about the contact-angle hysteresis, by comparing it with their numerical results Dimitrakopoulos and Higdon $^{6}$ found that this latter expression has only a very limited range of validity. Dimitrakopoulos and Higdon ${ }^{6}$ suggested relaxing the restriction on the contact-angle hysteresis, and did so in a limited manner by calculating the next order term in the asymptotic expansion of the critical value of the shear stress in the limit of small contact-angle hysteresis. In the present work we will remove this restriction and permit arbitrary contact-angle hysteresis consistent with the lubrication approximation.

The aim of the present work is to use the lubrication approximation to analyse three closely related problems involving a thin fluid rivulet or ridge (i.e. a two-dimensional droplet) subject to a prescribed uniform transverse shear stress at its free surface due to an external airflow, namely a rivulet draining under gravity down a vertical substrate, a rivulet driven by a longitudinal shear stress at its free surface, and a ridge on a horizontal substrate. Note that, unlike for a ridge, there has been very little work on a rivulet subject to a prescribed uniform transverse shear stress at its free surface. A rare example is the work of Darhuber et al. ${ }^{18}$ who used the lubrication approximation to study the deformation of and the mixing within a thin rivulet with pinned contact lines subject to a prescribed uniform temperature gradient (resulting in a prescribed uniform thermocapillary shear stress at its free surface). Like Dussan V. ${ }^{17}$ and Dimitrakopoulos and $\operatorname{Higdon}^{6}$ (Appendix) we use the lubrication approximation, but unlike them we do not place any further restriction on the contact-angle hysteresis, and thus are able to determine the deformation and de-pinning of thin rivulets and ridges for arbitrarily (small) contact angles. In particular, we explore situations in which both contact lines are pinned (as examined for a two-dimensional droplet by, for example, Schleizer and Bonnecaze ${ }^{8}$ and for a shear-driven rivulet by Darhuber et al. ${ }^{18}$ ) and in which de-pinning occurs at the advancing contact line (as examined for a two-dimensional droplet 
FIG. 1: Geometry of a gravity-driven rivulet on a vertical substrate subject to a prescribed transverse shear stress $\tau$.

by, for example, Dimitrakopoulos and Higdon ${ }^{6}$ ) or at the retreating contact line, as well as determining the critical "yield" condition (corresponding to de-pinning at both contact lines).

\section{A GRAVITY-DRIVEN RIVULET}

Consider the unsteady flow of a thin gravity-driven rivulet of fluid on a vertical substrate subject to a prescribed transverse shear stress $\tau$ at its free surface. Cartesian axes Oxyz are chosen with the $x$-axis vertically downwards, the $y$-axis parallel to the substrate $z=0$, and the $z$-axis normal to the substrate, and $\mathbf{g}=(g, 0,0)$ denotes acceleration due to gravity, as shown in Figure 1. Without loss of generality, we take $\tau \geq 0$ so that the shear stress acts from left to right in Figure 1. The fluid is assumed to be Newtonian with constant density $\rho$, viscosity $\mu$, and surface tension $\gamma$. The velocity $\mathbf{u}=(u(x, y, z, t), v(x, y, z, t), w(x, y, z, t))$ and pressure $p=p(x, y, z, t)$ of the fluid are governed by the familiar mass-conservation and Navier-Stokes equations subject to the usual normal and tangential stress balances and the kinematic condition at the free surface $z=h(x, y, t)$, and no-slip and no-penetration conditions at the substrate $z=0$. The positions of the contact lines are denoted by $y=$ $a_{1}(x, t)$ and $y=a_{2}(x, t)$, where $a_{1}<a_{2}$, so that $h\left(a_{1}\right)=h\left(a_{2}\right)=0$, and the rivulet has (small) contact angles $\beta_{1}=\beta_{1}(x, t)$ and $\beta_{2}=\beta_{2}(x, t)$ at $y=a_{1}$ and $y=a_{2}$, respectively, given by

$$
\beta_{1}=\left.\frac{h_{y}-a_{1 x} h_{x}}{\left[1+\left(a_{1 x}\right)^{2}\right]^{\frac{1}{2}}}\right|_{y=a_{1}}, \quad \beta_{2}=-\left.\frac{h_{y}-a_{2 x} h_{x}}{\left[1+\left(a_{2 x}\right)^{2}\right]^{\frac{1}{2}}}\right|_{y=a_{2}} .
$$

For most of the present work we will be concerned with equilibrium solutions which are independent of $x$ with parallel contact lines $a_{1}=a_{10}$ and $a_{2}=a_{20}$, constant contact angles $\beta_{1}=\beta_{10}$ and $\beta_{2}=\beta_{20}$, and free surface profile $h=h_{0}(y)$. In the general case $\tau>0$ we have $\beta_{10}<\beta_{20}$, while in the special case $\tau=0$ we have $\beta_{10}=\beta_{20}=\beta_{0}$, say. However, in Appendix A we will consider the quasi-static stability of these equilibrium solutions, and in that part of the work we will follow many previous authors (including Davis ${ }^{19}$, Weiland and 
Davis $^{20}$, and Young and Davis ${ }^{21}$ ) and assume that the normal velocities of the contact lines are related to their respective contact angles by the general "Tanner Laws"

$$
\frac{a_{1 t}}{\left[1+\left(a_{1 x}\right)^{2}\right]^{\frac{1}{2}}}=-\kappa F_{1}\left(\beta_{1}\right), \quad \frac{a_{2 t}}{\left[1+\left(a_{2 x}\right)^{2}\right]^{\frac{1}{2}}}=\kappa F_{2}\left(\beta_{2}\right)
$$

where $\kappa(>0)$ is an empirically determined constant with the dimensions of velocity. The dimensionless functions $F_{1}\left(\beta_{1}\right)$ and $F_{2}\left(\beta_{2}\right)$ satisfy $F_{1}\left(\beta_{10}\right)=0$ and $F_{2}\left(\beta_{20}\right)=0$, and are monotonically increasing near $\beta_{1}=\beta_{10}$ and $\beta_{2}=\beta_{20}$, respectively.

We non-dimensionalise according to

$$
\begin{gathered}
x=L x^{*}, \quad y=L y^{*}, \quad a_{1}=L a_{1}^{*}, \quad a_{2}=L a_{2}^{*}, \quad z=\beta_{0} L z^{*}, \quad h=\beta_{0} L h^{*}, \\
\beta_{1}=\beta_{0} \beta_{1}^{*}, \quad \beta_{2}=\beta_{0} \beta_{2}^{*}, \quad p-p_{\infty}=\frac{\beta_{0} \gamma}{L} p^{*}, \quad \tau=\frac{\beta_{0}^{2} \gamma}{L} \tau^{*}, \quad t=\frac{L}{\beta_{0} \kappa} t^{*}, \\
u=\frac{\beta_{0}^{3} \gamma}{\mu} u^{*}, \quad v=\frac{\beta_{0}^{3} \gamma}{\mu} v^{*}, \quad w=\frac{\beta_{0}^{4} \gamma}{\mu} w^{*},
\end{gathered}
$$

where $L=\beta_{0}^{1 / 2} l$ is a typical length scale in the $x$ and $y$ directions, and $p_{\infty}$ is the uniform atmospheric pressure, where $l=(\gamma / \rho g)^{1 / 2}$ is the capillary length. For clarity we immediately drop the star superscripts on non-dimensional variables.

At leading order in $\beta_{0} \ll 1$ the mass-conservation and Navier-Stokes equations are

$$
u_{x}+v_{y}+w_{z}=0
$$

and

$$
0=-p_{x}+1+u_{z z}, \quad 0=-p_{y}+v_{z z}, \quad 0=-p_{z},
$$

to be solved subject to conditions of no slip and no penetration at the substrate,

$$
u=v=w=0 \quad \text { on } \quad z=0,
$$

and balances of normal and tangential stress at the free surface,

$$
p=-\nabla^{2} h, \quad u_{z}=0 \quad \text { and } \quad v_{z}=\tau \quad \text { on } \quad z=h
$$

where $\nabla^{2}$ denotes the two-dimensional Laplacian. Solving (4) and (5) subject to (6) and (7) yields

$$
\begin{gathered}
p=-\nabla^{2} h, \quad u=\frac{p_{x}-1}{2}(z-2 h) z, \quad v=\frac{p_{y}}{2}(z-2 h) z+\tau z, \\
w=-\frac{\nabla^{2} p}{6}(z-3 h) z^{2}+\left[\left(p_{x}-1\right) h_{x}+p_{y} h_{y}\right] \frac{z^{2}}{2} .
\end{gathered}
$$


The kinematic free-surface condition can be written as

$$
C h_{t}+\nabla \cdot(\bar{u}, \bar{v})=0
$$

where $\bar{u}=\bar{u}(x, y, t)$ and $\bar{v}=\bar{v}(x, y, t)$ are the local fluxes in the $x$ and $y$ directions, respectively, namely

$$
\bar{u}=\int_{0}^{h} u \mathrm{~d} z=-\frac{\left(p_{x}-1\right) h^{3}}{3}, \quad \bar{v}=\int_{0}^{h} v \mathrm{~d} z=-\frac{p_{y} h^{3}}{3}+\frac{\tau h^{2}}{2},
$$

and $C=\mu \kappa / \beta_{0}^{2} \gamma$ is a capillary number. Hence the free surface profile $h$ satisfies the partial differential equation

$$
C h_{t}+\left(\frac{h^{3}}{3}\right)_{x}+\nabla \cdot\left(\frac{h^{3}}{3} \nabla \nabla^{2} h\right)+\left(\frac{\tau h^{2}}{2}\right)_{y}=0
$$

The longitudinal volume flux $Q_{\mathrm{g}}=Q_{\mathrm{g}}(x, t)$ through a transverse cross-section of the rivulet $x=$ constant is given by

$$
Q_{\mathrm{g}}=\int_{a_{1}}^{a_{2}} \bar{u} \mathrm{~d} y=-\int_{a_{1}}^{a_{2}} \frac{\left(p_{x}-1\right) h^{3}}{3} \mathrm{~d} y
$$

\section{A SHEAR-DRIVEN RIVULET AND A RIDGE}

The analysis in Section II concerns a gravity-driven rivulet on a vertical substrate, hereafter referred to simply as a "gravity-driven rivulet", but similar analyses apply to two other closely related problems, namely, a rivulet of fluid on a planar substrate driven by a prescribed longitudinal shear stress $T$, hereafter referred to simply as a "shear-driven rivulet", and a ridge of fluid on a horizontal substrate, hereafter referred to simply as a "ridge".

Proceeding as for the gravity-driven rivulet with $L=\beta_{0}^{2} \gamma / T$ for the shear-driven rivulet and $L$ left general for the ridge, but for simplicity restricting our attention to the case $L \ll l$ so that we may neglect the effect of gravity entirely, $p$ and $v$ are again given by (8) and $w$ is given by

$$
w=-\frac{\nabla^{2} p}{6}(z-3 h) z^{2}+\left(p_{x} h_{x}+p_{y} h_{y}\right) \frac{z^{2}}{2} .
$$

For the shear-driven rivulet

$$
u=\frac{p_{x}}{2}(z-2 h) z+z,
$$


and hence $h$ satisfies

$$
C h_{t}+\left(\frac{h^{2}}{2}\right)_{x}+\nabla \cdot\left(\frac{h^{3}}{3} \nabla \nabla^{2} h\right)+\left(\frac{\tau h^{2}}{2}\right)_{y}=0,
$$

and the longitudinal volume flux $Q_{\mathrm{s}}=Q_{\mathrm{s}}(x, t)$ through a transverse cross-section $x=$ constant is given by

$$
Q_{\mathrm{s}}=\int_{a_{1}}^{a_{2}} \bar{u} \mathrm{~d} y=\int_{a_{1}}^{a_{2}}-\frac{p_{x} h^{3}}{3}+\frac{h^{2}}{2} \mathrm{~d} y .
$$

For the ridge

$$
u=\frac{p_{x}}{2}(z-2 h) z
$$

and hence $h$ satisfies

$$
C h_{t}+\nabla \cdot\left(\frac{h^{3}}{3} \nabla \nabla^{2} h\right)+\left(\frac{\tau h^{2}}{2}\right)_{y}=0,
$$

and the area $A=A(x, t)$ of a transverse cross-section $x=$ constant is given by

$$
A=\int_{a_{1}}^{a_{2}} h \mathrm{~d} y .
$$

As a result of their strong similarities, in the remainder of this work we shall present results for all three problems in parallel, and, when results apply to all three, we simply refer to the "rivulet/ridge".

\section{EQUILIBRIUM SOLUTIONS}

As we have already mentioned, the rivulet/ridge has equilibrium solutions which are independent of $x$ with parallel contact lines $a_{1}=a_{10}$ and $a_{2}=a_{20}$ and constant contact angles $\beta_{1}=\beta_{10}$ and $\beta_{2}=\beta_{20}$. In this Section we describe the basic properties of these solutions (denoted with a subscript zero), and in order to do this it is convenient to choose the (arbitrary) location of the origin so that the contact lines are at $y= \pm a_{0}$, where $a_{0}$ is the semi-width of the rivulet/ridge. Hence for all three problems the free surface profile $h_{0}=h_{0}(y)$ satisfies the third-order nonlinear ordinary differential equation

$$
h_{0} h_{0}^{\prime \prime \prime}+\frac{3 \tau}{2}=0
$$

and the boundary conditions

$$
h_{0}\left( \pm a_{0}\right)=0, \quad h_{0}^{\prime}\left(-a_{0}\right)=\beta_{10}, \quad h_{0}^{\prime}\left(+a_{0}\right)=-\beta_{20},
$$

where a prime $\left(^{\prime}\right)$ denotes differentiation with respect to argument. 


\section{A. Local behaviour near the contact lines}

Local analysis of (20) near the contact lines reveals that when $\beta_{10}>0$ the free surface near the left-hand contact line behaves according to

$$
h_{0}=\beta_{10}\left(a_{0}+y\right)-\frac{3 \tau}{4 \beta_{10}}\left(a_{0}+y\right)^{2} \ln \left(a_{0}+y\right)+O\left(a_{0}+y\right)^{2}
$$

as $y \rightarrow-a_{0}^{+}$, and when $\beta_{20}>0$ the free surface near the right-hand contact line behaves according to

$$
h_{0}=\beta_{20}\left(a_{0}-y\right)+\frac{3 \tau}{4 \beta_{20}}\left(a_{0}-y\right)^{2} \ln \left(a_{0}-y\right)+O\left(a_{0}-y\right)^{2}
$$

as $y \rightarrow+a_{0}^{-}$, showing that $h_{0}^{\prime \prime}$ is, in general, logarithmically singular at both contact lines.

\section{B. Free surface profile}

Integrating (20) once and evaluating the resulting expression at $y=-a_{0}$ using (22) leads to

$$
h_{0} h_{0}^{\prime \prime}-\frac{1}{2}\left(h_{0}^{\prime 2}-\beta_{10}^{2}\right)+\frac{3 \tau}{2}\left(y+a_{0}\right)=0 .
$$

In particular, at any stationary point of $h_{0}$, denoted by $h_{0}=h_{\mathrm{m}}$ at $y=y_{\mathrm{m}}$, where $-a_{0}<$ $y_{\mathrm{m}}<+a_{0}$, we have $h_{0}^{\prime}=0$, so that from (24) we have

$$
h_{\mathrm{m}} h_{0}^{\prime \prime}\left(y_{\mathrm{m}}\right)=-\frac{1}{2} \beta_{10}^{2}-\frac{3 \tau}{2}\left(y_{\mathrm{m}}+a_{0}\right)<0
$$

Thus we deduce that any stationary point must be a maximum, and hence that the free surface profile of the rivulet/ridge must always have a single maximum $h_{0}=h_{\mathrm{m}}$ at $y=y_{\mathrm{m}}$.

\section{Transverse force balance}

Evaluating (24) at $y=a_{0}$ using (23) yields an important relationship between the contact angles $\beta_{10}$ and $\beta_{20}$, the rivulet/ridge semi-width $a_{0}$ and the shear stress $\tau$, namely

$$
\beta_{20}^{2}-\beta_{10}^{2}=6 \tau a_{0}
$$

Physically (26) represents a transverse balance of forces due to capillary and shear-stress effects. 


\section{Longitudinal velocity}

In equilibrium $p_{0 x}=0$, and hence from (8), (14) and (17) the longitudinal velocity $u_{0}$ is given by $u_{0}=\left(2 h_{0}-z\right) z / 2$ for the gravity-driven rivulet (with maximum velocity $u_{0}=h_{\mathrm{m}}^{2} / 2$ at $y=y_{\mathrm{m}}$ and $z=h_{\mathrm{m}}$ ), $u_{0}=z$ for the shear-driven rivulet (with maximum velocity $u_{0}=h_{\mathrm{m}}$ at $y=y_{\mathrm{m}}$ and $z=h_{\mathrm{m}}$ ), and $u_{0} \equiv 0$ for the ridge, respectively.

From (12) the longitudinal volume flux of a gravity-driven rivulet is

$$
Q_{\mathrm{g}}=\frac{1}{3} \int_{-a_{0}}^{+a_{0}} h_{0}^{3} \mathrm{~d} y,
$$

from (16) the longitudinal volume flux of a shear-driven rivulet is

$$
Q_{\mathrm{s}}=\frac{1}{2} \int_{-a_{0}}^{+a_{0}} h_{0}^{2} \mathrm{~d} y
$$

while from (19) the cross-sectional area of a ridge is

$$
A=\int_{-a_{0}}^{+a_{0}} h_{0} \mathrm{~d} y .
$$

\section{E. Transverse velocities}

In equilibrium $p_{0 x}=0$ and $p_{0 y}=3 \tau / 2 h_{0}$, and hence from (8) and (13) the transverse velocities $v_{0}$ and $w_{0}$ are given by

$$
v_{0}=\frac{\tau}{4 h_{0}}\left(3 z-2 h_{0}\right) z, \quad w_{0}=\frac{\tau h_{0}^{\prime}}{4 h_{0}^{2}} z^{3}
$$

therefore the stream function of the transverse flow $\psi_{0}=\psi_{0}(y, z)$, defined by $v_{0}=\psi_{0 z}$, $w_{0}=-\psi_{0 y}$ and $\psi_{0}=0$ on $z=0$, is given by

$$
\psi_{0}=\frac{\tau}{4 h_{0}}\left(z-h_{0}\right) z^{2}
$$

in agreement with the corresponding analytical results given by King and Tuck ${ }^{15}$ (their Eq. (A1)) and Darhuber et al. ${ }^{18}$ (their Eq. (4.2) and (4.3)), and with the numerical results obtained by Dimitrakopoulos and $\operatorname{Higdon}^{6}$ (their Fig. 4). At any stagnation point of the transverse flow we have $v_{0}=w_{0}=0$, implying from (30) that either $z=0$ or $z=2 h_{0} / 3$ and $h_{0}^{\prime}=0$, and hence ( since $h_{0}$ always has a single maximum $h_{0}=h_{\mathrm{m}}$ at $y=y_{\mathrm{m}}$ ) that the transverse flow always has a single interior stagnation point at $y=y_{\mathrm{m}}$ and $z=2 h_{\mathrm{m}} / 3$. 
The clockwise transverse "circulation flux" about this stagnation point is given by $-\psi_{0}$ evaluated at the stagnation point, and hence is equal to $\tau h_{\mathrm{m}}^{2} / 27(>0)$. Note that for both a gravity-driven and a shear-driven rivulet (but not, of course, for a ridge) the combination of longitudinal and transverse velocities means that fluid particles spiral (clockwise) along the rivulet in a helical manner, as described by Darhuber et al. ${ }^{18}$ for a rivulet subject to a prescribed uniform shear stress at its free surface.

\section{F. Solutions with prescribed volume flux or area}

Thus far the discussion has been for an equilibrium rivulet/ridge with general values of $\beta_{10}, \beta_{20}, \tau$ and $a_{0}$ satisfying (26) whose longitudinal volume flux or area is given by (27), (28) or (29), respectively. However, in order to investigate the effect of varying the transverse shear stress $\tau$ in a systematic way it is sensible to consider a rivulet/ridge with prescribed flux/area as $\tau$ is increased from zero. Physically we may interpret this as the quasi-equilibrium development of a rivulet/ridge with prescribed flux/area as the shear stress is slowly increased from zero. Prescribing the values of the shear stress $\tau$ and the flux/area means that one of the remaining three quantities $\beta_{10}, \beta_{20}$ and $a_{0}$ must also be prescribed. The most physically sensible way to do this is firstly to prescribe the semi-width $a_{0}$ (corresponding to a rivulet/ridge with two pinned contact lines and with the unknown contact angles $\beta_{10}$ and $\beta_{20}$ determined as part of the solution), then to prescribe $\beta_{10}$ or $\beta_{20}$ (corresponding to a rivulet/ridge with one pinned and one de-pinned contact line and with the unknown semi-width $a_{0}$ and the other contact angle determined as part of the solution), and finally to prescribe $\beta_{10}$ and $\beta_{20}$ (corresponding to the critical "yield" condition beyond which no equilibrium solutions are possible).

In the special case of no transverse shear stress, $\tau=0$, the rivulet/ridge has the familiar parabolic free surface profile $h_{0}=H_{0}(y)$ given by

$$
H_{0}=\frac{a_{0}^{2}-y^{2}}{2 a_{0}}
$$

with maximum height $h_{\mathrm{m}}=a_{0} / 2$ at $y=0$ and equal contact angles $\beta_{10}=\beta_{20}=1$, and (27)-(29) yield

$$
Q_{\mathrm{g}}=\frac{4 a_{0}^{4}}{105}, \quad Q_{\mathrm{s}}=\frac{2 a_{0}^{3}}{15}, \quad A=\frac{2 a_{0}^{2}}{3},
$$


respectively. For simplicity of presentation in what follows we choose (without loss of generality) the prescribed flux/area values to be

$$
Q_{\mathrm{g}}=\frac{4}{105}, \quad Q_{\mathrm{s}}=\frac{2}{15}, \quad A=\frac{2}{3},
$$

corresponding to setting $a_{0}=1$ in the appropriate expressions in the case $\tau=0$ given by (33). Note, however, that prescribing $Q_{\mathrm{g}}, Q_{\mathrm{s}}$ and $A$ according to (34) does not guarantee that $a_{0}=1$ for all $\tau>0$; indeed determining when and how $a_{0}$ varies as $\tau$ is varied is one of the key issues discussed in Sections V and VI.

In Appendix A we show that an equilibrium ridge/rivulet with prescribed flux/area is quasi-statically stable to two-dimensional perturbations.

\section{PINNED SOLUTIONS WITH PRESCRIBED SEMI-WIDTH}

In this Section we describe the development of the equilibrium rivulet/ridge solutions with prescribed semi-width $a_{0}=1$ but varying $\beta_{10}$ and $\beta_{20}$ as $\tau$ is increased from zero. Physically we may interpret these solutions as a rivulet/ridge with pinned (i.e. fixed) contact lines but varying contact angles. Note that the results obtained in this Section are in qualitative agreement with those of Schleizer and Bonnecaze ${ }^{8}$ for a two-dimensional droplet in a shear flow, and with those of Darhuber et al. ${ }^{18}$ for a rivulet subject to a prescribed uniform shear stress at its free surface.

\section{A. Limit of small transverse shear stress, $\tau \rightarrow 0^{+}$}

In the limit of small transverse shear stress, $\tau \rightarrow 0^{+}$, the free surface profile $h_{0}=h_{0}(y)$ takes the form $h_{0}=H_{0}(y)+\tau H_{1}(y)+O\left(\tau^{2}\right)$, where $H_{0}$ is given by (32) and $H_{1}$ satisfies

$$
H_{1}^{\prime \prime \prime}=-\frac{3}{1-y^{2}}
$$

subject to the fixed-contact-line conditions

$$
H_{1}( \pm 1)=0
$$

and the prescribed flux/area condition

$$
\int_{-1}^{+1} H_{0}^{n-1} H_{1} \mathrm{~d} y=0
$$


where $n=3$ for a gravity-driven rivulet, $n=2$ for a shear-driven rivulet and $n=1$ for a ridge. Solving (35) subject to (36) and (37) shows that, rather unexpectedly, $H_{1}$ is the same for all three problems, namely

$$
H_{1}=\frac{3}{4}\left[(1-y)^{2} \ln (1-y)-(1+y)^{2} \ln (1+y)+4 y \ln 2\right]
$$

In particular, (38) shows that $H_{1}<0$ when $-1<y<0, H_{1}>0$ when $0<y<1$,

$$
\begin{aligned}
\beta_{10} & =1-\frac{3}{2} \tau+O\left(\tau^{2}\right) \\
\beta_{20} & =1+\frac{3}{2} \tau+O\left(\tau^{2}\right) \\
h_{\mathrm{m}} & =\frac{1}{2}+\frac{9}{8}(2 \ln 2-1)^{2} \tau^{2}+O\left(\tau^{4}\right) \\
y_{\mathrm{m}} & =\frac{3}{2}(2 \ln 2-1) \tau+O\left(\tau^{3}\right)
\end{aligned}
$$

revealing that the effect of a small transverse shear stress is to push the rivulet/ridge down on the left and up on the right, i.e. to skew the rivulet/ridge to the right.

\section{B. General case of non-zero transverse shear stress, $\tau>0$}

In the general case of non-zero transverse shear stress, $\tau>0$, the rivulet/ridge is nonsymmetric with $0<\beta_{10}<\beta_{20}$ and the free surface profile is obtained by solving (20) subject to (21) and the prescribed flux/area condition numerically. This was done by converting the problem into an initial value problem by using the local behaviour of $h_{0}$ either near $y=-a_{0}$ given by (22) or near $y=a_{0}$ given by (23) to generate approximate initial conditions which were imposed close to (but not at) the appropriate contact line. For example, using (22) yields the approximate initial conditions

$$
\begin{aligned}
& h_{0}\left(-a_{0}+\delta\right)=\beta_{10} \delta-\frac{3 \tau}{4 \beta_{10}} \delta^{2} \ln \delta+k \delta^{2}, \\
& h_{0}^{\prime}\left(-a_{0}+\delta\right)=\beta_{10}-\frac{3 \tau}{4 \beta_{10}}(2 \delta \log \delta+\delta)+2 k \delta, \\
& h_{0}^{\prime \prime}\left(-a_{0}+\delta\right)=-\frac{3 \tau}{4 \beta_{10}}(2 \log \delta+3)+2 k,
\end{aligned}
$$

where $\delta \ll 1$ was chosen to be sufficiently small (typically $\delta=10^{-6}$ ) and $k$ is a free parameter. Solutions were then obtained by iterating $\tau$ and $k$ for a given value of $\beta_{10}$ until the conditions 
FIG. 2: Free surface profiles $z=h_{0}(y)$ of a gravity-driven rivulet for $\tau=0,0.08,0.16,0.24,0.32$ and $\tau_{\max } \simeq 0.3646$. The corresponding profiles for a shear-driven rivulet and for a ridge are qualitatively similar.

FIG. 3: Plot of (a) the contact angles $\beta_{10}$ and $\beta_{20}$, (b) the maximum height of the rivulet/ridge $h_{\mathrm{m}}$, and (c) the location of the maximum height of the rivulet/ridge $y_{\mathrm{m}}$, as functions of $\tau$ for each of the three problems.

of zero height at the other contact line, $h\left(a_{0}\right)=0$, and of prescribed flux/area were satisfied to within an appropriate tolerance (typically $10^{-6}$ ). The consistency of the numerical results obtained was checked by substituting the values of $\beta_{10}, \beta_{20}, \tau$ and $a_{0}$ into the transverse balance of forces $(26)$.

Figure 2 shows numerically calculated free surface profiles of a gravity-driven rivulet with $a_{0}=1$ for various values of $\tau$, illustrating how the profile becomes increasingly skewed to the right as $\tau$ is increased from zero. The corresponding profiles for a shear-driven rivulet and for a ridge are qualitatively similar and hence are omitted for brevity. Figure 3 shows how the contact angles $\beta_{10}$ and $\beta_{20}$, the maximum height $h_{\mathrm{m}}$, and the location of the maximum height $y_{\mathrm{m}}$, vary with $\tau$. In particular, Figure 3 shows that for all three problems $\beta_{20}(>1)$, $h_{\mathrm{m}}(>1 / 2)$ and $y_{\mathrm{m}}(>0)$ increase monotonically while $\beta_{10}(<1)$ decreases monotonically (with, of course, $a_{0}=1$ ) as $\tau$ is increased from zero. Figure 3 also shows that there is a maximum value of $\tau$, denoted by $\tau_{\max }$, at which $\beta_{10}=0$, and hence from (26) at which $\beta_{20}=\beta_{\max }=\left(6 \tau_{\max }\right)^{1 / 2}$, beyond which no equilibrium solution with prescribed semi-width $a_{0}=1$ is possible. Table I gives the values of $\tau_{\max }$ and the corresponding maximum values of $\beta_{20}=\beta_{\max }, h_{\mathrm{m}}$ and $y_{\mathrm{m}}$ when $\tau=\tau_{\max }$ for each of the three problems.

\section{DE-PINNED SOLUTIONS WITH VARIABLE SEMI-WIDTH}

Thus far we have considered only pinned contact lines; however, a contact line will not, in general, remain pinned for all values of its contact angle, and typically there is a finite range of possible equilibrium contact angles, denoted by $\beta_{\mathrm{R}} \leq \beta \leq \beta_{\mathrm{A}}$, where $\beta_{\mathrm{A}}$ and $\beta_{\mathrm{R}}$ are the so-called advancing and receding contact angles, respectively. As, for example, Dussan V. ${ }^{17}$ and Blake and Ruschak ${ }^{22}$ describe, the advancing angle $\beta_{\mathrm{A}}$ is the largest value that $\beta$ can take before the contact line begins to advance, the receding angle $\beta_{\mathrm{R}}$ is the smallest value 


\begin{tabular}{|c||c|c|c|}
\hline & Gravity-driven Rivulet & Shear-driven Rivulet & Ridge \\
\hline \hline$\tau_{\max }$ & 0.3646 & 0.3730 & 0.3924 \\
\hline$\beta_{\max }$ & 1.4791 & 1.4959 & 1.5344 \\
\hline$h_{\mathrm{m}}$ & 0.5149 & 0.5208 & 0.5342 \\
\hline$y_{\mathrm{m}}$ & 0.1962 & 0.1962 & 0.1962 \\
\hline
\end{tabular}

TABLE I: Values of $\tau_{\max }$ and the corresponding maximum values of $\beta_{20}=\beta_{\max }, h_{\mathrm{m}}$ and $y_{\mathrm{m}}$ when $\tau=\tau_{\max }$ for each of the three problems.

that $\beta$ can take before the contact line begins to recede, and the values of $\beta_{\mathrm{A}}$ and $\beta_{\mathrm{R}}$ depend on the properties of the fluid and of the substrate. This means that, in practice, one or both of the contact lines will de-pin before the maximum value $\tau=\tau_{\max }$ is reached. Hence in this Section we extend the analysis presented in Section $\mathrm{V}$ for equilibrium rivulets/ridges with prescribed semi-width $a_{0}=1$ but varying contact angles to equilibrium rivulets/ridges with varying semi-width $a_{0} \neq 1$ but one or two prescribed contact angles, namely $\beta_{10}=\beta_{\mathrm{R}}$ and/or $\beta_{20}=\beta_{\mathrm{A}}$. Specifically, in the next three Subsections we consider rivulets/ridges that de-pin only at the advancing (i.e. right-hand) contact line, only at the receding (i.e. left-hand) contact line, and at both contact lines, respectively.

In what follows we denote the critical value of $\tau$ at which de-pinning first occurs, i.e. the critical value of $\tau$ at which the solution with prescribed semi-width described in Section $\mathrm{V}$ predicts that either $\beta_{10}=\beta_{\mathrm{R}}$ or $\beta_{20}=\beta_{\mathrm{A}}$, by $\tau=\tau_{\text {depin }}$. When $\tau<\tau_{\text {depin }}$ the solutions with prescribed semi-width $a_{0}=1$ described in Section $\mathrm{V}$ still apply, but when $\tau>\tau_{\text {depin }}$ we solve (20) subject to (21) and the prescribed flux/area condition with either $\beta_{10}=\beta_{\mathrm{R}}$ or $\beta_{20}=\beta_{\mathrm{A}}$ prescribed, as appropriate, and calculate values of the semi-width, $a_{0} \neq 1$, and the other contact angle, $\beta_{10}$ or $\beta_{20}$, as part of the solution. In these latter calculations it is again convenient to choose the (arbitrary) location of the origin so that the contact lines are at $y= \pm a_{0}$ (where, in general, $a_{0} \neq 1$ ); however, since the location of the origin is arbitrary, when $\tau>\tau_{\text {depin }}$ we may interpret the de-pinned solutions with the pinned contact line at either $y=-1$ or $y=1$, as appropriate. 
FIG. 4: Free surface profiles $z=h_{0}(y)$ of a gravity-driven rivulet with advancing contact angle $\beta_{20}=$ $\beta_{\mathrm{A}}=1.3$ for $\tau=\tau_{\text {depin }} \simeq 0.2208,0.2266,0.2324,0.2382,0.2440,0.2498$ and $\tau_{\text {depinmax }} \simeq 0.2557$. The corresponding profiles for a shear-driven rivulet and for a ridge are qualitatively similar.

FIG. 5: Plot of (a) the semi-width $a_{0}$, (b) the contact angles $\beta_{10}$ and $\beta_{20}$, (c) the maximum height of the rivulet/ridge $h_{\mathrm{m}}$, and (d) the location of the maximum height of the rivulet/ridge $y_{\mathrm{m}}$, for a rivulet/ridge with advancing contact angle $\beta_{\mathrm{A}}=1.3$ as a function of $\tau$ for each of the three problems. The three vertical dashed lines indicate the appropriate values of $\tau_{\text {depin }}$.

\section{A. De-pinning Only at the Advancing Contact Line}

To illustrate a rivulet/ridge that de-pins only at the advancing (i.e. right-hand) contact line we choose $\beta_{\mathrm{A}}=1.3$ and $\beta_{\mathrm{R}}=0$, but note that any other value of $\beta_{\mathrm{A}}$ satisfying $1<$ $\beta_{\mathrm{A}}<\beta_{\max }$ will give qualitatively similar results. Table II gives the values of $\tau_{\text {depin }}$ and the corresponding values of $\beta_{10}, h_{\mathrm{m}}$ and $y_{\mathrm{m}}$ when $\tau=\tau_{\text {depin }}$ for each of the three problems in the case $\beta_{\mathrm{A}}=1.3$. Note that the results obtained in this Subsection are in qualitative agreement with those obtained by Dimitrakopoulos and Higdon ${ }^{6}$ for a two-dimensional droplet in a shear flow.

Figure 4 shows the free surface profiles $z=h_{0}(y)$ of a gravity-driven rivulet for various values of $\tau \geq \tau_{\text {depin }} \simeq 0.2208$ illustrating how the profile is further skewed to the right, and is flattened and widened as $\tau$ is increased from $\tau_{\text {depin }}$. The corresponding profiles for a shear-driven rivulet and for a ridge are qualitatively similar and hence are omitted for brevity.

Figure 5 shows how the semi-width $a_{0}$, the contact angles $\beta_{10}$ and $\beta_{20}$, the maximum height $h_{\mathrm{m}}$, and the location of the maximum height $y_{\mathrm{m}}$, vary with $\tau$. In particular, this figure shows that for all three problems $a_{0}(>1)$ and $y_{\mathrm{m}}$ increase monotonically while $\beta_{10}$ and $h_{\mathrm{m}}$ decrease monotonically (with, of course, $\beta_{20}=\beta_{\mathrm{A}}$ ) as $\tau$ is increased from $\tau_{\text {depin }}$. Figure 5 also shows that, as in the case of a rivulet/ridge with two pinned contact lines, there is a maximum value of $\tau$, denoted now by $\tau_{\text {depinmax }}$, at which $\beta_{10}=0$, and hence from (26) at which $a_{0}=a_{0 \max }=\beta_{\mathrm{A}}^{2} /\left(6 \tau_{\text {depinmax }}\right)$, beyond which no equilibrium solutions with prescribed advancing contact angle $\beta_{20}=\beta_{\mathrm{A}}$ is possible. Table III gives the values of 


\begin{tabular}{|c||c|c|c|}
\hline & Gravity-driven Rivulet & Shear-driven Rivulet & Ridge \\
\hline \hline$\tau_{\text {depin }}$ & 0.2208 & 0.2187 & 0.2149 \\
\hline$\beta_{10}$ & 0.6041 & 0.6145 & 0.6330 \\
\hline$h_{\mathrm{m}}$ & 0.5054 & 0.5070 & 0.5099 \\
\hline$y_{\mathrm{m}}$ & 0.1236 & 0.1217 & 0.1183 \\
\hline
\end{tabular}

TABLE II: Values of $\tau_{\text {depin }}$ and the corresponding values of $\beta_{10}, h_{\mathrm{m}}$ and $y_{\mathrm{m}}$ when $\tau=\tau_{\text {depin }}$ for each of the three problems in the case $\beta_{\mathrm{A}}=1.3$.

\begin{tabular}{|c||c|c|c|}
\hline & Gravity-driven Rivulet & Shear-driven Rivulet & Ridge \\
\hline \hline$\tau_{\text {depinmax }}$ & 0.2557 & 0.2565 & 0.2593 \\
\hline$a_{0 \max }$ & 1.1016 & 1.0981 & 1.0864 \\
\hline$h_{\mathrm{m}}$ & 0.4986 & 0.4970 & 0.4917 \\
\hline$y_{\mathrm{m}}$ & 0.3178 & 0.3136 & 0.2996 \\
\hline
\end{tabular}

TABLE III: Values of $\tau_{\text {depinmax }}$ and the corresponding values of $a_{0}=a_{0 \max }, h_{\mathrm{m}}$ and $y_{\mathrm{m}}$ when $\tau=\tau_{\text {depinmax }}$ for each of the three problems in the case $\beta_{\mathrm{A}}=1.3$.

$\tau_{\text {depinmax }}$ and the corresponding values of $a_{0}=a_{0 \max }, h_{\mathrm{m}}$ and $y_{\mathrm{m}}$ when $\tau=\tau_{\text {depinmax }}$ for each of the three problems in the case $\beta_{\mathrm{A}}=1.3$. Note that $a_{0}$ and $y_{\mathrm{m}}$ attain their maximum values given in Table III at $\tau=\tau_{\text {depinmax }}$, while $h_{\mathrm{m}}$ attains its maximum value given in Table II at $\tau=\tau_{\text {depin }}$. Furthermore, note that for all three problems the values of $\tau_{\max }$ given in Table I and the corresponding values of $\tau_{\text {depinmax }}$ given in Table III satisfy $\tau_{\text {depinmax }}<\tau_{\max }$, i.e. a rivulet/ridge that has de-pinned at the advancing contact line cannot exist for as large a transverse shear stress as the corresponding one with two pinned contact lines.

\section{B. De-pinning Only at the Receding Contact Line}

To illustrate a rivulet/ridge that de-pins only at the receding (i.e. left-hand) contact line we choose $\beta_{\mathrm{R}}=0.5$ and $\beta_{\mathrm{A}}=\infty$, but note that any other value of $\beta_{\mathrm{R}}$ satisfying $0<\beta_{\mathrm{R}}<1$ will give qualitatively similar results. Table IV gives the values of $\tau_{\text {depin }}$ and the corresponding values of $\beta_{20}, h_{\mathrm{m}}$ and $y_{\mathrm{m}}$ when $\tau=\tau_{\text {depin }}$ for each of the three problems in 
FIG. 6: Free surface profiles $z=h_{0}(y)$ of a gravity-driven rivulet with receding contact angle $\beta_{10}=\beta_{\mathrm{R}}=0.5$ for $\tau=\tau_{\text {depin }} \simeq 0.2635,0.3,0.4,0.5, \ldots, 1.7,1.8,2,5,10,15,20$ and 25 . The corresponding profiles for a shear-driven rivulet and for a ridge are qualitatively similar.

FIG. 7: Plot of (a) the semi-width $a_{0}$, (b) the contact angles $\beta_{10}$ and $\beta_{20}$, (c) the maximum height of the rivulet/ridge $h_{\mathrm{m}}$, and (d) the location of the maximum height of the rivulet/ridge $y_{\mathrm{m}}$, for a rivulet/ridge with receding contact angle $\beta_{\mathrm{R}}=0.5$ as a function of $\tau$ for each of the three problems. In part (a) the leading order asymptotic behaviours of $a_{0}$ in the limit of large transverse shear stress $\tau \rightarrow \infty$, given by $a_{0} \sim 0.7594 \tau^{-3 / 11}$ for a gravity-driven rivulet, $a_{0} \sim 0.7815 \tau^{-1 / 4}$ for a shear-driven rivulet, and $a_{0} \sim 0.8294 \tau^{-1 / 5}$ for a ridge, are shown with dashed lines. The three vertical dashed lines (which are impossible to distinguish in part (a)) indicate the appropriate values of $\tau_{\text {depin }}$.

the case $\beta_{\mathrm{R}}=0.5$.

Figure 6 shows the free surface profiles $z=h_{0}(y)$ of a gravity-driven rivulet for various values of $\tau \geq \tau_{\text {depin }} \simeq 0.2635$ illustrating how the profile is again further skewed to the right but (in contrast to the case of a rivulet that de-pins only at the advancing contact line) is thickened and narrowed as $\tau$ is increased from $\tau_{\text {depin }}$. The corresponding profiles for a shear-driven rivulet and for a ridge are qualitatively similar and hence are omitted for brevity.

Figure 7 shows how the semi-width $a_{0}$, the contact angles $\beta_{10}$ and $\beta_{20}$, the maximum height $h_{\mathrm{m}}$, and the location of the maximum height $y_{\mathrm{m}}$, vary with $\tau$. In particular, this figure shows that for all three problems $\beta_{20}, h_{\mathrm{m}}$ and $y_{\mathrm{m}}$ increase monotonically while $a_{0}(<1)$ decreases monotonically (with, of course, $\beta_{10}=\beta_{\mathrm{R}}$ ) as $\tau$ is increased from $\tau_{\text {depin. }}$. Figure 7 also shows that an equilibrium solution with a prescribed receding contact angle $\beta_{10}=\beta_{\mathrm{R}}$ is possible for all values of $\tau>\tau_{\text {depin }}$ (i.e. there is no maximum value of $\tau$ corresponding to $\tau_{\text {depinmax }}$ in Subsection VIA). In particular, this means that, unlike in the cases of a rivulet/ridge with two pinned contact lines and of a rivulet/ridge that de-pins only at the advancing contact line, a rivulet/ridge that de-pins only at the receding contact line can exist for an arbitrarily large transverse shear stress.

In the limit of large transverse shear stress, $\tau \rightarrow \infty$, the numerical solutions shown in Figure 7 suggest that the rivulet/ridge becomes infinitely narrow like $a_{0}=O\left(\tau^{-\frac{n}{3 n+2}}\right) \rightarrow 0^{+}$ 


\begin{tabular}{|c||c|c|c|}
\hline & Gravity-driven Rivulet & Shear-driven Rivulet & Ridge \\
\hline \hline$\tau_{\text {depin }}$ & 0.2635 & 0.2674 & 0.2756 \\
\hline$\beta_{20}$ & 1.3532 & 1.3617 & 1.3797 \\
\hline$h_{\mathrm{m}}$ & 0.5076 & 0.5104 & 0.5163 \\
\hline$y_{\mathrm{m}}$ & 0.1457 & 0.1462 & 0.1472 \\
\hline
\end{tabular}

TABLE IV: Values of $\tau_{\text {depin }}$ and the corresponding values of $\beta_{20}, h_{\mathrm{m}}$ and $y_{\mathrm{m}}$ when $\tau=\tau_{\text {depin }}$ for each of the three problems in the case $\beta_{\mathrm{R}}=0.5$.

and infinitely thick like $h_{\mathrm{m}}=O\left(\tau^{\frac{1}{3 n+2}}\right) \rightarrow \infty$, and so we seek an asymptotic solution in the form

$$
\begin{gathered}
h_{0}=\tau^{\frac{1}{3 n+2}} \bar{h}_{0}, \quad y=\tau^{-\frac{n}{3 n+2}} \bar{y}, \quad z=\tau^{\frac{1}{3 n+2}} \bar{z} \\
a_{0}=\tau^{-\frac{n}{3 n+2}} \bar{a}_{0}, \quad \beta_{20}=\tau^{\frac{n+1}{3 n+2}} \bar{\beta}_{20}, \quad h_{\mathrm{m}}=\tau^{\frac{1}{3 n+2}} \bar{h}_{\mathrm{m}}, \quad y_{\mathrm{m}}=\tau^{-\frac{n}{3 n+2}} \bar{y}_{\mathrm{m}}
\end{gathered}
$$

where $n$ has the same meaning as in Section V A. At leading order in the limit $\tau \rightarrow \infty(20)$ and (21) reduce to

$$
\bar{h}_{0} \bar{h}_{0}^{\prime \prime \prime}+\frac{3}{2}=0
$$

subject to

$$
\bar{h}_{0}\left( \pm \bar{a}_{0}\right)=0, \quad \bar{h}_{0}^{\prime}\left(-\bar{a}_{0}\right)=0, \quad \bar{h}_{0}^{\prime}\left(+\bar{a}_{0}\right)=-\bar{\beta}_{20},
$$

and the prescribed flux/area condition, which was solved numerically using the same approach as that employed to solve (20) subject to (21) and the prescribed flux/area condition, but using the appropriate local behaviour near the left-hand contact line, namely

$$
h_{0}=2 \sqrt{\tau}\left(a_{0}+y\right)^{\frac{3}{2}}+O\left(a_{0}+y\right)^{\frac{5+\sqrt{13}}{4}}
$$

as $y \rightarrow-a_{0}^{+}$in place of (22), to yield the solutions for the scaled free surface profile $\bar{z}=\bar{h}_{0}(\bar{y})$ and the values of $\bar{a}_{0}, \bar{\beta}_{20}, \bar{h}_{\mathrm{m}}$ and $\bar{y}_{\mathrm{m}}$ given in Table V. Figure 8 shows the scaled free surface profile $\bar{z}=\bar{h}_{0}(\bar{y})$ of a gravity-driven rivulet. The corresponding results for a shear-driven rivulet and for a ridge are qualitatively similar and hence are omitted for brevity. Figure 7(a) includes the leading order asymptotic behavour of $a_{0}$ in the limit $\tau \rightarrow \infty$ given by $a_{0} \sim \tau^{-\frac{n}{3 n+2}} \bar{a}_{0}$, and shows that the asymptotic results are in good agreement with the exact 
FIG. 8: Scaled free surface profile $\bar{z}=\bar{h}_{0}(\bar{y})$ of a gravity-driven rivulet obtained by solving (47) subject to (48) and the prescribed flux condition and relevant to a rivulet that de-pins only at its receding contact line in the limit of large transverse shear stress $\tau \rightarrow \infty$. The corresponding results for a shear-driven rivulet and for a ridge are qualitatively similar.

\begin{tabular}{|c||c|c|c|}
\hline & Gravity-driven Rivulet & Shear-driven Rivulet & Ridge \\
\hline \hline $\bar{a}_{0}$ & 0.7594 & 0.7815 & 0.8294 \\
\hline $\bar{\beta}_{20}$ & 2.1346 & 2.1654 & 2.2307 \\
\hline $\bar{h}_{\mathrm{m}}$ & 0.5644 & 0.5891 & 0.6441 \\
\hline $\bar{y}_{\mathrm{m}}$ & 0.1490 & 0.1533 & 0.1627 \\
\hline
\end{tabular}

TABLE V: Values of $\bar{a}_{0}, \bar{\beta}_{20}, \bar{h}_{\mathrm{m}}$ and $\bar{y}_{\mathrm{m}}$ obtained by solving (47) subject to (48) and the prescribed flux/area condition for each of the three problems and relevant to a rivulet/ridge that de-pins only at its receding contact line in the limit of large shear stress $\tau \rightarrow \infty$.

ones even for relatively small values of $\tau$. Of course, in practice, the present solution will eventually fail at a large but finite value of $\tau$ because either $\beta_{20}$ becomes so large that the advancing contact line de-pins or $a_{0}$ becomes so small and/or $h_{\mathrm{m}}$ becomes so large that the original assumption that the rivulet/ridge is thin breaks down.

\section{De-pinning at Both Contact Lines}

Except in the cases $\beta_{\mathrm{R}}=0$ and $\beta_{\mathrm{A}}=\infty$ considered in Subsections VIA and VIB, respectively, in general as $\tau$ is increased from zero depinning will eventually occur at both contact lines. Beyond this critical yield value of $\tau$, denoted by $\tau_{\text {yield }}$, no equilibrium solution exists and the rivulet/ridge will evolve unsteadily. From $(26), \tau_{\text {yield }}$ and the corresponding critical yield value of $a$, denoted by $a_{\text {yield }}$, are related by

$$
\beta_{\mathrm{A}}^{2}-\beta_{\mathrm{R}}^{2}=6 a_{\text {yield }} \tau_{\text {yield }}
$$

which coincides with the thin-film limit of the corresponding result given by Dimitrakopoulos and Higdon ${ }^{6}$ for a ridge (their Eq. (A2)). In the special case of small contact-angle hysteresis 


\begin{tabular}{|c||c|c|c|}
\hline & Gravity-driven Rivulet & Shear-driven Rivulet & Ridge \\
\hline \hline$\tau_{\text {yield }}$ & 0.2340 & 0.2338 & 0.2342 \\
\hline$a_{\text {yield }}$ & 1.0259 & 1.0264 & 1.0250 \\
\hline$h_{\mathrm{m}}$ & 0.5029 & 0.5032 & 0.5025 \\
\hline$y_{\mathrm{m}}$ & 0.1460 & 0.1461 & 0.1458 \\
\hline
\end{tabular}

TABLE VI: Values of $\tau_{\text {yield }}$ and the corresponding values of $a_{\text {yield }}, h_{\mathrm{m}}$ and $y_{\mathrm{m}}$ when $\tau=\tau_{\text {yield }}$ for each of the three problems in the case $\beta_{\mathrm{R}}=0.5$ and $\beta_{\mathrm{A}}=1.3$.

in which $\beta_{\mathrm{A}} \simeq \beta_{\mathrm{R}} \simeq 1, \Delta \beta=\beta_{\mathrm{A}}-\beta_{\mathrm{R}} \ll 1$ and $a_{\text {yield }} \simeq a_{0}$ equation (50) gives

$$
\tau_{\text {yield }} \simeq \frac{\Delta \beta}{3 a_{0}} \ll 1 \text {. }
$$

In particular, for a ridge (51) reduces to the corresponding result obtained by Dimitrakopoulos and Higdon ${ }^{6}$ in this special case (their Eq. (A4)). However, in general, the contact-angle hysteresis will not be small (i.e. $\beta_{\mathrm{A}}$ and $\beta_{\mathrm{R}}$ will not be close to unity and $a_{\text {yield }}$ will not be close to $a_{0}$ ) and the values of $\tau_{\text {yield }}$ and $a_{\text {yield }}$ have to be determined from the numerical results already obtained in Subsections VIA and VIB by identifying the value of $\tau$ (or, equivalently, the value of $a$ ) at which $\beta_{10}=\beta_{\mathrm{R}}$ in Subsection VI A or $\beta_{20}=\beta_{\mathrm{A}}$ in Subsection VIB, as appropriate. Table VI gives the values of $\tau_{\text {yield }}$ and the corresponding values of

$a_{\text {yield }}, h_{\mathrm{m}}$ and $y_{\mathrm{m}}$ when $\tau=\tau_{\text {yield }}$ for each of the three problems in the case $\beta_{\mathrm{R}}=0.5$ and $\beta_{\mathrm{A}}=1.3$.

\section{CONCLUSIONS}

In the present work we used the lubrication approximation to analyse three closely related problems involving a thin rivulet or ridge of fluid subject to a prescribed uniform transverse shear stress $\tau$ at its free surface due to an external airflow, namely a rivulet draining under gravity down a vertical substrate, a rivulet driven by a longitudinal shear stress at its free surface, and a ridge on a horizontal substrate, and found qualitatively similar behaviour for all three problems.

In Section IV we described the general properties of equilibrium rivulet/ridge solutions with parallel contact lines $a_{1}=a_{10}$ and $a_{2}=a_{20}$ and constant contact angles $\beta_{1}=\beta_{10}$ 
and $\beta_{2}=\beta_{20}$. In particular, we showed that the free surface profile of the equilibrium rivulet/ridge always has a single maximum and that the transverse flow within it always has a single internal stagnation point.

In Sections V and VI we described the quasi-equilibrium development of solutions with prescribed flux/area as $\tau$ is varied. In Section $\mathrm{V}$ we showed that the free surface profile of a rivulet/ridge with pinned contact lines is skewed to the right as $\tau$ is increased from zero, and that there is a maximum value of $\tau=\tau_{\max }$ (corresponding to $\beta_{10}=0$ ) beyond which no solution with prescribed semi-width is possible. In practice, one or both of the contact lines will de-pin before the maximum value $\tau=\tau_{\max }$ is reached, and so in Section VI we considered a rivulet/ridge that de-pins at one or both contact lines. In particular, we determined the critical value of $\tau=\tau_{\text {depin }}\left(<\tau_{\max }\right)$ at which de-pinning first occurs. For $\tau>\tau_{\text {depin }}$ the free surface profile of the rivulet/ridge is further skewed to the right, but otherwise the behaviour is qualitatively different for rivulets/ridges that first depin at the advancing or at the receding contact line. In the case of de-pinning only at the advancing contact line the rivulet/ridge is flattened and widened as $\tau$ is increased from $\tau_{\text {depin }}$, and there is a second maximum value $\tau=\tau_{\text {depinmax }}\left(<\tau_{\max }\right)$ (again corresponding to $\beta_{10}=0$ ) beyond which no solution with a prescribed advancing contact angle $\beta_{20}=\beta_{\mathrm{A}}$ is possible. In contrast, in the case of de-pinning only at the receding contact line the rivulet/ridge is thickened and narrowed as $\tau$ is increased from $\tau_{\text {depin }}$, and a solution with a prescribed receding contact angle $\beta_{10}=\beta_{\mathrm{R}}$ is possible for all values of $\tau>\tau_{\text {depin }}$. In general, in the case of de-pinning at both contact lines there is a critical yield value of the shear stress $\tau=\tau_{\text {yield }}$ beyond which no equilibrium solution is possible and the rivulet/ridge will evolve unsteadily.

In Appendix A we showed that an equilibrium rivulet/ridge with prescribed flux/area is quasi-statically stable to two-dimensional perturbations.

Although not considered in the present work, the unsteady evolution of the rivulet/ridge is also of considerable interest. Indeed, an example of precisely this situation has already been analysed by Smith $^{23}$ who studied the thermocapillary-driven motion of a thin twodimensional droplet on a substrate with a prescribed uniform temperature gradient. In the special case of a droplet with zero heat loss at its free surface and no slip at the substrate, 
Smith's ${ }^{23}$ problem is mathematically equivalent to the unsteady version of the present ridge

problem. Smith investigated the particular case $\beta_{\mathrm{A}}=1$ and $\beta_{\mathrm{R}}=0.8$ and found that as the non-dimensional temperature gradient (equivalent to the present $\tau$ ) increases from zero the droplet immediately de-pins at the advancing contact line (because $\beta_{\mathrm{A}}=1$ ) followed by de-pinning at the retreating contact line and eventually by non-existence of equilibrium solutions. This behaviour is both consistent with the present results and in accord with our physical expectations and, based on the results of the present work, we would expect qualitatively similar behaviour for both the gravity-driven and the shear-driven rivulet problems.

\section{Acknowledgements}

The first and second authors (JMS and CP) gratefully acknowledge the financial support of the United Kingdom Engineering and Physical Sciences Research Council (EPSRC) via a Doctoral Training Account (DTA) studentship and of the University of Strathclyde via a Postgraduate Research Scholarship, respectively. This work was completed while the corresponding author (SKW) was a Visiting Fellow in the Department of Mechanical and Aerospace Engineering, School of Engineering and Applied Science, Princeton University, USA, and a Visiting Fellow in the Oxford Centre for Collaborative Applied Mathematics (OCCAM), Mathematical Institute, University of Oxford, United Kingdom. This publication was based on work supported in part by Award No KUK-C1-013-04, made by King Abdullah University of Science and Technology (KAUST).

\section{Appendix A: Quasi-Static Stability of an Equilibrium Ridge/Rivulet}

In this Appendix we show that an equilibrium ridge/rivulet with prescribed flux/area is quasi-statically stable to two-dimensional perturbations.

At leading order in the limit of small capillary number, $C \rightarrow 0$, equation (9) becomes simply $\nabla \cdot(\bar{u}, \bar{v})=0$ so that the rivulet/ridge is quasi-static and evolves according to the general Tanner Laws (2).

Following the analysis described in Sections II - IV the free surface profile $z=h(y)$ of a 
two-dimensional quasi-static rivulet/ridge satisfies

$$
h h^{\prime \prime \prime}+\frac{3 \tau}{2}=0
$$

subject to the contact-line conditions

$$
h\left(a_{1}\right)=h\left(a_{2}\right)=0
$$

and the prescribed flux/area condition

$$
\frac{1}{n} \int_{a_{1}}^{a_{2}} h^{n} \mathrm{~d} y=\text { constant }
$$

in which the prescribed values of the flux/area are given by (33) and $n$ has the same meaning as in Section VA. The contact angles $\beta_{1}=\beta_{1}(t)$ and $\beta_{2}=\beta_{2}(t)$ are given by $\beta_{1}=h^{\prime}\left(a_{1}\right)$ and $\beta_{2}=-h^{\prime}\left(a_{2}\right)$, and from the general Tanner Laws (2) the positions of the contact lines at $a_{1}=a_{1}(t)$ and $a_{2}=a_{2}(t)$ evolve according to

$$
a_{1 t}=-F_{1}\left(\beta_{1}\right), \quad a_{2 t}=F_{2}\left(\beta_{2}\right) .
$$

The semi-width of the rivulet/ridge, denoted by $a=a(t)$, is given by $a=\left(a_{2}-a_{1}\right) / 2$, and, since the location of the origin is arbitrary, we note that the two-dimensional quasi-static solution depends on $a_{1}$ and $a_{2}$ only in the combination $a$.

We can investigate the quasi-static (and, in general, nonlinear) stability of any of the equilibrium rivulet/ridge solutions $h=h_{0}$ described previously with semi-width $a=a_{0}$ and contact angles $\beta_{1}=\beta_{10}$ and $\beta_{2}=\beta_{20}$ by seeking a perturbed solution in the form $h=h_{0}+h_{1}$ with perturbed positions of the contact lines $a_{1}=-a_{0}+a_{11}$ and $a_{2}=a_{0}+a_{21}$, and hence perturbed semi-width $a=a_{0}+\hat{a}_{1}$, where $\hat{a}_{1}=\left(a_{21}-a_{11}\right) / 2$, and perturbed contact angles $\beta_{1}=\beta_{10}+\beta_{11}$ and $\beta_{2}=\beta_{20}+\beta_{21}$. At leading order in the perturbation quantities, the Tanner Laws (A4) reduce to

$$
a_{11 t}=-\frac{M}{m !} \beta_{11}^{m}, \quad a_{21 t}=\frac{N}{n !} \beta_{21}^{n},
$$

where $M$ and $N$, defined by

$$
\begin{gathered}
M=\left.\frac{\mathrm{d}^{m} F_{1}}{\mathrm{~d} \beta_{1}^{m}}\right|_{\beta_{1}=\beta_{10}}>0, \quad m=1,3,5, \ldots, \\
N=\left.\frac{\mathrm{d}^{n} F_{2}}{\mathrm{~d} \beta_{2}^{n}}\right|_{\beta_{2}=\beta_{20}}>0, \quad n=1,3,5, \ldots,
\end{gathered}
$$


are the first non-zero derivatives of $F_{1}$ and $F_{2}$ (of orders $m$ and $n$, respectively) at $\beta_{1}=\beta_{10}$ and $\beta_{2}=\beta_{20}$, respectively. The fact that the solution depends on $a_{1}$ and $a_{2}$ only in the combination $a=\left(a_{2}-a_{1}\right) / 2$ means that

$$
\beta_{11}=\left[a_{11} \frac{\partial \beta_{1}}{\partial a} \frac{\partial a}{\partial a_{1}}+a_{21} \frac{\partial \beta_{1}}{\partial a} \frac{\partial a}{\partial a_{2}}\right]_{\substack{a_{1}=-a_{0} \\ a_{2}=a_{0}}}=\left.\frac{\left(a_{21}-a_{11}\right)}{2} \frac{\partial \beta_{1}}{\partial a}\right|_{a=a_{0}}=\left.\hat{a}_{1} \frac{\partial \beta_{1}}{\partial a}\right|_{a=a_{0}}
$$

with a similar expression for $\beta_{21}$, and hence (A5) can be expressed as

$$
a_{11 t}=-\frac{M}{m !}\left(\alpha_{1} \hat{a}_{1}\right)^{m}, \quad a_{21 t}=\frac{N}{n !}\left(\alpha_{2} \hat{a}_{1}\right)^{n}
$$

and therefore

$$
\hat{a}_{1 t}=\frac{1}{2}\left[\frac{M}{m !}\left(\alpha_{1} \hat{a}_{1}\right)^{m}+\frac{N}{n !}\left(\alpha_{2} \hat{a}_{1}\right)^{n}\right]
$$

where

$$
\alpha_{1}=\left.\frac{\partial \beta_{1}}{\partial a}\right|_{a=a_{0}}, \quad \alpha_{2}=\left.\frac{\partial \beta_{2}}{\partial a}\right|_{a=a_{0}} .
$$

In practice, it is likely that $m=n$ and $M=N$, and in this case we can solve (A10) and hence (A9) to yield

$$
a_{11}(t)=a_{11}(0)-\frac{\hat{a}_{1}(0) M \alpha_{1}^{m}}{\sigma} \times \begin{cases}e^{\sigma t}-1 & \text { if } m=1, \\ \left(\frac{(1-m) \sigma t}{m ! \hat{a}_{1}(0)^{1-m}}+1\right)^{\frac{1}{1-m}}-1 & \text { if } m=3,5,7, \ldots,\end{cases}
$$

with a similar solution for $a_{21}(t)$, where

$$
\sigma=\frac{M}{2}\left(\alpha_{1}^{m}+\alpha_{2}^{m}\right)
$$

and $\hat{a}_{1}(0)=\left[a_{21}(0)-a_{11}(0)\right] / 2$ is the initial perturbation to the semi-width of the rivulet/ridge. Hence perturbations grow exponentially (if $m=n=1$ ) or algebraically (if $m=n=3,5,7, \ldots$ ) when $\sigma>0$ and decay when $\sigma<0$, and so we deduce that an equilibrium rivulet/ridge is stable when $\sigma<0$ and unstable when $\sigma>0$. Thus in order to determine the quasi-static stability of the rivulet/ridge we need to calculate the values of $\alpha_{1}$ and $\alpha_{2}$ defined by (A11). Solving (A1) subject to (A2) and (A3) numerically using the same approach as that employed to solve (20) subject to (21) and the prescribed flux/area condition reveals that $\alpha_{1}<0$ and $\alpha_{2}<0$ for all values of $\tau$, and hence we deduce that 
an equilibrium rivulet/ridge is unconditionally quasi-statically stable to two-dimensional perturbations.

Sullivan $^{24}$ also showed that an equilibrium rivulet/ridge with pinned contact lines is linearly stable to three-dimensional perturbations.

1 T. G. Myers and J. P. F. Charpin, "A mathematical model for atmospheric ice accretion and water flow on a cold surface," Int. J. Heat Mass Transf. 47, 5483 (2004).

2 F.-C. Chou and P.-Y. Wu, "Effect of air shear on film planarization during spin coating," J. Electrochem. Soc. 147, 699 (2000).

3 A. C. Robertson, I. J. Taylor, S. K. Wilson, B. R. Duffy, and J. M. Sullivan, "Numerical simulation of rivulet evolution on a horizontal cable subject to an external aerodynamic field," J. Fluids Struct. 26, 50 (2010).

4 J. Fan, M. C. T. Wilson, and N. Kapur, "Displacement of liquid droplets on a surface by a shearing air flow," J. Coll. Int. Sci. 356, 286 (2011).

5 X. Li and C. Pozrikidis, "Shear flow over a liquid drop adhering to a solid surface," J. Fluid Mech. 307, 167 (1996).

6 P. Dimitrakopoulos and J. J. L. Higdon, "Displacement of fluid droplets from solid surfaces in low-Reynolds-number shear flows," J. Fluid Mech. 336, 351 (1997).

7 P. Dimitrakopoulos and J. J. L. Higdon, "On the displacement of three-dimensional fluid droplets from solid surfaces in low-Reynolds-number shear flows," J. Fluid Mech. 377, 189 (1998).

8 A. D. Schleizer and R. T. Bonnecaze, "Displacement of a two-dimensional immiscible droplet adhering to a wall in shear and pressure-driven flows," J. Fluid Mech. 383, 29 (1999).

9 S. Yon and C. Pozrikidis, "Deformation of a liquid drop adhering to a plane wall: significance of the drop viscosity and the effect of an insoluble surfactant," Phys. Fluids 11, 1297 (1999).

10 P. Dimitrakopoulos, "Deformation of a droplet adhering to a solid surface in shear flow: onset of interfacial sliding," J. Fluid Mech. 580, 451 (2007).

11 J. Zhang, M. J. Miksis, and S. G. Bankoff, "Nonlinear dynamics of a two-dimensional viscous drop under shear flow," Phys. Fluids 18, 072106 (2006).

12 P. D. M. Spelt, "Shear flow past two-dimensional droplets pinned or moving on an adhering 
channel wall at moderate Reynolds numbers: a numerical study," J. Fluid Mech. 561, 439 (2006).

13 H. Ding and P. D. M. Spelt, "Onset of motion of a three-dimensional droplet on a wall in shear flow at moderate Reynolds numbers," J. Fluid Mech. 599, 341 (2008).

14 H. Ding, M. N. H. Gilani, and P. D. M. Spelt, "Sliding, pinch-off and detachment of a droplet on a wall in a shear flow," J. Fluid Mech. 644, 217 (2010).

15 A. C. King and E. O. Tuck, "Thin liquid layers supported by steady air-flow surface traction," J. Fluid Mech. 251, 709 (1993).

16 K. Sugiyama and M. Sbragaglia, "Linear shear flow past a hemispherical droplet adhering to a solid surface," J. Eng. Maths 62, 35 (2008).

17 E. B. Dussan V., "On the ability of drops to stick to surfaces of solids. Part 3. The influences of the motion of the surrounding fluid on dislodging drops," J. Fluid Mech. 174, 381 (1987).

18 A. A. Darhuber, J. Z. Chen, J. M. Davis, and S. M. Troian, "A study of mixing in thermocapillary flows on micropatterned surfaces," Phil. Trans. R. Soc. Lond. A 362, 1037 (2004).

19 S. H. Davis, "Moving contact lines and rivulet instabilities. Part 1. The static rivulet," J. Fluid Mech. 98, 225 (1980).

20 R. H. Weiland and S. H. Davis, "Moving contact lines and rivulet instabilities. Part 2. Long waves on flat rivulets," J. Fluid Mech. 107, 261 (1981).

21 G. W. Young and S. H. Davis, "Rivulet instabilities," J. Fluid Mech. 176, 1 (1987).

22 T. D. Blake and K. J. Ruschak, "Wetting: static and dynamic contact lines," Chapter 3 in "Liquid Film Coating". Eds S. F. Kistler and P. M. Schweizer, (Chapman and Hall, London, 1997), pp. 63-97.

23 M. K. Smith, "Thermocapillary migration of a two-dimensional liquid droplet on a solid surface," J. Fluid Mech. 294, 209 (1995).

24 J. M. Sullivan, "Thin-film flows subject to an external shear stress", $\mathrm{Ph} \mathrm{D}$ Thesis, University of Strathclyde, Glasgow, United Kingdom (2008). 